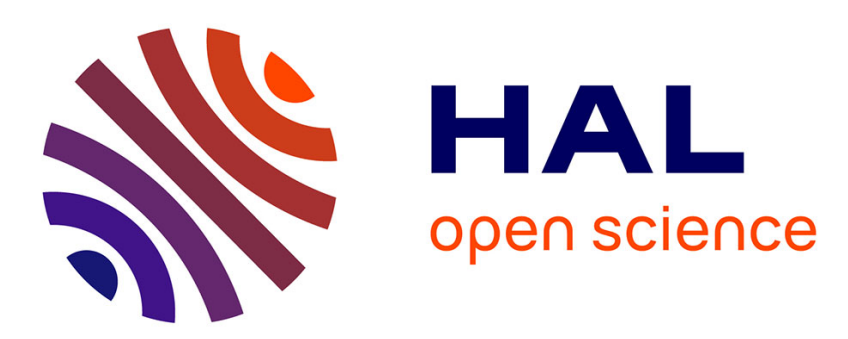

\title{
A New Method for Limb Singularity Analysis of Parallel Manipulators
}

\author{
Semaan Amine, Ossama Mokhiamar, Stéphane Caro
}

\section{To cite this version:}

Semaan Amine, Ossama Mokhiamar, Stéphane Caro. A New Method for Limb Singularity Analysis of Parallel Manipulators. International Journal of Robotics and Automation, 2019, 34 (3), 10.2316/J.2019.206-5494 . hal-02404161

\section{HAL Id: hal-02404161 https://hal.science/hal-02404161}

Submitted on 11 Dec 2019

HAL is a multi-disciplinary open access archive for the deposit and dissemination of scientific research documents, whether they are published or not. The documents may come from teaching and research institutions in France or abroad, or from public or private research centers.
L'archive ouverte pluridisciplinaire HAL, est destinée au dépôt et à la diffusion de documents scientifiques de niveau recherche, publiés ou non, émanant des établissements d'enseignement et de recherche français ou étrangers, des laboratoires publics ou privés. 


\title{
A NEW METHOD FOR LIMB SINGULARITY ANALYSIS OF PARALLEL MANIPULATORS
}

\author{
Semaan Amine \\ Department of Mechanical Engineering \\ Beirut Arab University \\ P.O. Box 115020 \\ Beirut, Lebanon \\ email:s.amin@bau.edu.lb \\ Ossama Mokhiamar \\ Department of Mechanical Engineering \\ Alexandria University \\ El-Chatby, Alexandria 21544 \\ Alexandria, Egypt \\ email: usamam@alexu.edu.eg \\ Stéphane Caro
}

RoMas - Robots and Machines for Manufacturing, Society and Services

LS2N - Laboratoire des Sciences du Numérique de Nantes

CNRS - Centre National de la Recherche Scientifique

1, rue de la Noë, BP 92101, 44321 NANTES Cedex 3

Nantes, France

email: stephane.caro@1s2n.fr

\begin{abstract}
This paper presents a new method for analyzing the limb singularities of lower-mobility parallel manipulators using the superbracket of Grassmann-Cayley algebra. For lower-mobility limbs, the limb twist system does not form a $6 \times$ 6 matrix. The proposed method allows forming a Jacobian matrix, and thus, a superbracket for any lower-mobility limb by adding twists that correspond to the limb's constrained motion(s). The method is applied to four- and five-degrees
\end{abstract}

of freedom limbs.

\section{Key Words}

limb singularity, screw theory, GrassmannCayley algebra, superbracket

\section{Introduction}

Lower-mobility Parallel Manipulators (PMs) have been a center of attraction for research in the field of robot kinematics for the last two 
decades [1-4], due to their wide range of applications and low power consumption as compared to Stewart platforms. Limb singularities [5] are configurations of PMs in which the moving platform loses some degree(s) of freedom (DOFs). The characterization of limb singularities is important since they affect the overall motion of the manipulator. These singularities are related to the degeneracy of a limb twist system, namely, the screw system spanned by the set of twist screws of a limb kinematic joints. They can be determined analytically $[6,7]$ by examining the rank deficiency of the matrix associated with the limb twist system. In general, the analytical procedure requires long hand calculations, does not easily lead to simple geometric and vector conditions for limb singularities, and cannot enumerate all possible limb singularities.

In Grassmann-Cayley algebra, the superbracket corresponds to the determinant of a Jacobian matrix, namely, a $6 \times 6$ matrix formed by the coordinate vectors of six Plücker lines. The superbracket decomposition has been known in the past decade for its effectiveness in the singularity analysis of PMs. It was first applied by Ben-Horin et al. [8] to solve for the parallel singularities of Gough Stewart PMs and then used to analyze the parallel singularities of lowermobility PMs $[9,10]$. It allows finding vector expressions and geometric conditions for the vanishing conditions of the determinant of a Jacobian matrix. However, the superbracket decomposition has not been used for limb singularity analysis yet.

For a 6 -DOF limb, the superbracket can be easily written from the $6 \times 6$ kinematic Jacobian matrix composed of the six twists of the limb. However, for lower-mobility limbs, namely, limbs with less than six DOFs, the limb twist system is a screw system (screw space) of dimension less than six and hence, it is not possible to form a $6 \times 6$ kinematic Jacobian matrix uniquely from the limb twists. Therefore, this paper defines a new technique that allows writing a $6 \times 6$ kinematic Jacobian matrix for any lower-mobility limb, which enables the use of the superbracket decomposition to enumerate the singularity conditions of lower-mobility limbs. To the best of the authors' knowledge, such an analysis is investigated for the first time in this paper.

The remainder of the paper is organized as follows. First, some fundamental concepts are recalled. Second, a technique to construct a kinematic Jacobian matrix for lower-mobility limbs is defined. Limb singularities are then studied by examining the rank deficiency of the constructed limb kinematic Jacobian matrix using the superbracket decomposition. The proposed method is explained through the analysis of limb singularities for the Orthoglide and the 4-RUU PM. The singular configurations are expressed in both algebraic and vector forms and are illustrated as well. Finally, the effectiveness and soundness of the proposed method are demonstrated through a comparison with the screw-theory-based method.

\section{Fundamentals}

\subsection{Grassmann-Cayley Algebra and Pro- jective Space}

The 3-dimensional projective space is constructed by adding a plane at infinity $\mathcal{P}_{\infty}$ to the 3-dimensional real space. In Grassmann-Cayley algebra, geometric entities are called extensors and are represented by Plücker coordinates of vectors.

A point $P$ in the 3 -dimensional real space is represented in Grassmann-Cayley algebra by an extensor $\mathrm{p}$ of step 1 and corresponds to a finite point in the 3-dimensional projective space. Each projective point has four Plücker coordinates. A line $P Q$ in the 3-dimensional real space is represented in Grassmann-Cayley algebra by an extensor pq of step 2 and corresponds to a finite line in the 3-dimensional projective space. Each projective line has six Plücker coordinates. For example, the finite projective line pq can 
be written as ${ }^{1}: \mathrm{pq}=\left[\begin{array}{c}\mathbf{s} \\ \mathbf{r}_{P} \times \mathbf{s}\end{array}\right]$, where $\mathbf{s}$ is the unit vector along line $P Q$. Each finite line in the 3-dimensional projective space has a unique point at infinity. Points at infinity are represented by underlined extensors. For instance, line $P Q$ can be represented by the extensor pq or by the extensor $\underline{p} \underline{r}$ where $\underline{\underline{r}}=\left[\begin{array}{l}\mathbf{s} \\ 0\end{array}\right]$. A plane of normal vector $\mathbf{n}$ in the 3-dimensional real space has a unique line at infinity, which can be represented by its Plücker coordinate vector $\left[\begin{array}{c}\mathbf{0}_{1 \times 3} \\ \mathbf{n}\end{array}\right]$.

\subsection{Superbracket and its Decomposition}

The superbracket corresponds to the determinant of a $6 \times 6$ matrix formed by the coordinate vectors of six Plücker lines. Thus, this superbracket vanishes whenever the six Plücker lines become linearly dependent. The superbracket involves the extensors of 12 projective points, i.e., two points on each Plücker line. The superbracket can be decomposed into a linear combination of 24 bracket monomials [11], where a bracket monomial is a product of three brackets and, in turn, a bracket corresponds to the determinant of a $4 \times 4$ matrix obtained from the Plücker coordinates of four projective points. The superbracket decomposition is given by:

$$
[\mathrm{ab} c d \text { ef } \mathrm{gh} i \mathrm{j} \mathrm{kl}]=\sum_{i=1}^{24} y_{i}
$$

\footnotetext{
${ }^{1}$ In this paper, the position vector of a point $P$ is denoted as $\mathbf{r}_{P}$
}

where

$$
\begin{aligned}
& y_{1}=-[\mathrm{abcd}][\mathrm{efgi}][\mathrm{hjkl}] \quad y_{2}=[\mathrm{abcd}][\mathrm{efhi}][\mathrm{gjkl}] \\
& y_{3}=[\mathrm{abcd}][\mathrm{efgj}][\mathrm{hikl}] \quad y_{4}=-[\mathrm{abcd}][\mathrm{efhj}][\mathrm{gikl}] \\
& y_{5}=[\mathrm{abce}][\mathrm{dfgh}][\mathrm{ijkl}] \quad y_{6}=-[\mathrm{abde}][\mathrm{cfgh}][\mathrm{ijkl}] \\
& y_{7}=-[\mathrm{abcf}][\mathrm{degh}][\mathrm{ijkl}] \quad y_{8}=[\mathrm{abdf}][\mathrm{cegh}][\mathrm{i} j \mathrm{kl}] \\
& y_{9}=-[\mathrm{abce}][\mathrm{dghi}][\mathrm{fjkl}] \quad y_{10}=[\mathrm{abde}][\mathrm{cghi}][\mathrm{fjkl}] \\
& y_{11}=[\mathrm{abcf}][\mathrm{dghi}][\mathrm{ejkl}] \quad y_{12}=[\mathrm{abce}][\operatorname{dgh} j][\mathrm{fikl}] \\
& y_{13}=-[\operatorname{abdf}][\mathrm{cghi}][\mathrm{ejkl}] \quad y_{14}=-[\mathrm{abde}][\mathrm{cghj}][\mathrm{fikl}] \\
& y_{15}=-[a b c f][\operatorname{dghj}][e i k l] \quad y_{16}=[a b d f][c g h j][e i k l] \\
& y_{17}=[\mathrm{abcg}][\operatorname{def} \mathrm{i}][\mathrm{hj} \mathrm{kl}] \quad y_{18}=-[\mathrm{abdg}][\text { cef } \mathrm{i}][\mathrm{hjkl}] \\
& y_{19}=-[a b c h][\operatorname{def} i][g j k l] \quad y_{20}=-[a b c g][\operatorname{def} j][h i k l] \\
& y_{21}=[\mathrm{abdh}][\text { cefi }][\mathrm{gjkl}] \quad y_{22}=[\mathrm{abdg}][\text { cefj] }[\text { hikl }] \\
& y_{23}=[\mathrm{abch}][\operatorname{def} j][\text { gikl }] \\
& y_{24}=-[\text { abdh }][\text { cefj] }[\text { gikl }]
\end{aligned}
$$

A bracket $[a b c d]$ vanishes if and only if (iff) the projective points $a, b, c$ and $d$ are coplanar.

\subsection{Screw Theory}

Screw theory $[12,13]$ provides an efficient tool for the type synthesis and the analysis of the instantaneous motion of manipulators. A twist is a screw that represents the instantaneous motion of a rigid body. A unit twist is denoted as $\hat{\epsilon}$. A zero-pitch twist corresponds to a pure rotational motion and takes the form $\hat{\epsilon}_{0}=\left[\begin{array}{c}\mathbf{s} \\ \mathbf{r}_{P} \times \mathbf{s}\end{array}\right], \mathbf{s}$ and $P$ being the direction of, and a point on the screw axis, respectively. In turn, an infinite-pitch twist corresponds to a pure translational motion and takes the form $\hat{\epsilon}_{\infty}=\left[\begin{array}{c}\mathbf{0}_{1 \times 3} \\ \mathbf{p}\end{array}\right], \mathbf{p}$ being a unit vector along the direction of translation.

\subsection{Singularities}

Lower-mobility PMs can exhibit several types of singularities, for instance: limb singularities, actuation singularities, and constraint singularities. A limb singularity corresponds to the degeneracy of a limb twist system and is characterized by the loss of some $\mathrm{DOF}(\mathrm{s})$ of the moving platform. In an actuation singularity, the PM will have some uncontrollable motions, namely, the actuators momentarily lose the ability to control the motion of the moving platform. In a constraint singularity, the lower-mobility PM loses some constraint(s) and, as a result, gains some $\mathrm{DOF}(\mathrm{s})$. 


\section{Limb Singularities and Kinematic Jacobian Matrix}

A limb singularity is characterized by a certain degeneracy in the limb kinematic screw system, namely, the limb twist system. The main objective of this paper is to enable the construction of a $6 \times 6$ kinematic Jacobian matrix for any PM limb and the examination of limb singularities by using the superbracket decomposition.

\subsection{Kinematic Jacobian Matrix of 6-DOF Limbs}

The twist system of a 6 -DOF limb is a screw system of dimension 6 . For such a limb, a $6 \times 6$ kinematic Jacobian matrix can be constructed from the limb twists.

\subsection{Kinematic Jacobian Matrix of Lower- Mobility Limbs}

Unlike 6-DOF limbs, the twist system of a lowermobility limb with $n<6$-DOF is a screw system of dimension $n$ and cannot form a $6 \times 6$ Jacobian matrix. Here, the main contribution of this paper is presented. Indeed, a concept is defined to write a $6 \times 6$ kinematic Jacobian matrix for any lowermobility limb.

Definition: A kinematic Jacobian matrix for a limb with $n<6-D O F$ can be constructed by adding, to the $n$ limb twists, a set of $6-n$ linearly independent twist(s) that correspond(s) to the constrained motion(s) of the limb.

In the following sections, the proposed concept is applied to analyze the limb singularities of the Orthoglide robot and the 4-RUU PM.

\section{Singularity Analysis of a 4-DOF PR $\Pi R$ Limb}

\subsection{Orthoglide Robot}

Fig. 1 shows a CAD model of the Orthoglide [14], a 3-DOF translational PM. Each limb of the Orthoglide is a PR $\Pi \mathrm{R}$ 3T1R limb, i.e., provides three independent translations and one rotation about an axis of fixed direction.

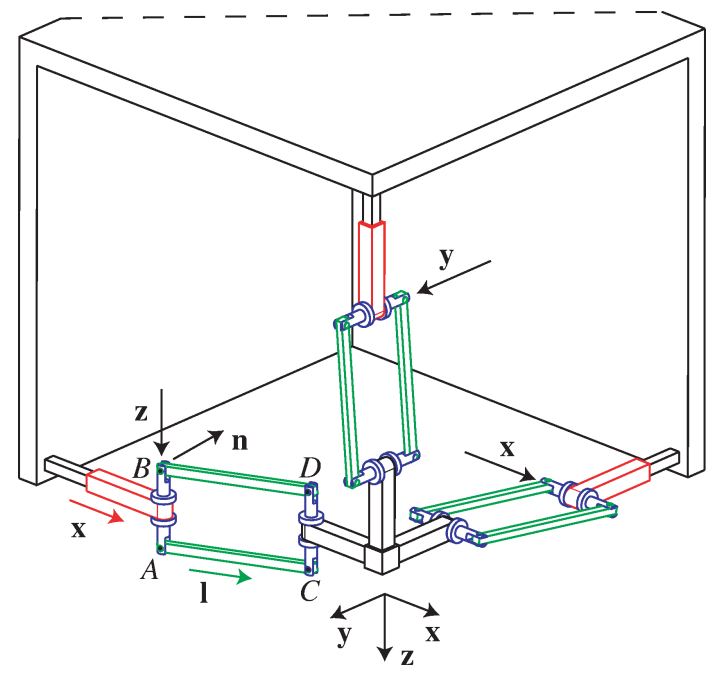

Figure 1: CAD model of the Orthoglide

\subsection{Orthoglide Limb Description}

Fig. 2 shows a limb of the Orthoglide. It is a 4DOF limb composed of four kinematic joints. The first one is a prismatic joint directed along vector $\mathbf{x}$. The second one is a revolute joint whose axis is directed along vector $\mathbf{z}$ and passes through point $A$. The third one is a planar parallelogram joint, named $\Pi$-joint [15], generating a circular translation along vector $\mathbf{I} \times \mathbf{n}$, where $\mathbf{I}$ is the unit vector along line $A C$ and $\mathbf{n}$ is the unit vector normal to the plane of the parallelogram $A B D C$. Note that vectors $\mathbf{n}$ and $\mathbf{z}$ are orthogonal to each other. The fourth joint is a revolute joint whose axis is directed along $\mathbf{z}$ and passes through point $C$. Accordingly, the PR $\Pi \mathrm{R} \operatorname{limb}$ twists can be written as follows: $\hat{\epsilon}_{\infty 1}=\left[\begin{array}{l}\mathbf{0} \\ \mathbf{x}\end{array}\right], \hat{\epsilon}_{01}=$ $\left[\begin{array}{c}\mathbf{z} \\ \mathbf{r}_{A} \times \mathbf{z}\end{array}\right], \hat{\epsilon}_{\infty 2}=\left[\begin{array}{c}\mathbf{0} \\ \mathbf{l} \times \mathbf{n}\end{array}\right]$, and $\hat{\epsilon}_{02}=\left[\begin{array}{c}\mathbf{z} \\ \mathbf{r}_{C} \times \mathbf{z}\end{array}\right]$.

\section{3. $\quad$ PR $\Pi$ R Limb Twist System}

Let $\mathrm{a}$ and $\mathrm{c}$ be the extensors of step 1 representing the Plücker coordinate vectors of the finite points $A$ and $C$, respectively. In addition, let $\underline{\mathrm{x}}=\left[\begin{array}{l}\mathbf{x} \\ 0\end{array}\right], \underline{\mathrm{y}}=$ $\left[\begin{array}{l}\mathbf{y} \\ 0\end{array}\right], \underline{\mathbf{z}}=\left[\begin{array}{l}\mathbf{z} \\ 0\end{array}\right], \underline{\underline{I}}=\left[\begin{array}{l}\mathbf{l} \\ 0\end{array}\right]$, and $\underline{\underline{n}}=\left[\begin{array}{l}\mathbf{n} \\ 0\end{array}\right]$ represent the points at infinity of any finite line along vectors $\mathbf{x}, \mathbf{y}$, $\mathbf{z}, \mathbf{l}$, and $\mathbf{n}$, respectively. Thus, $\hat{\epsilon}_{\infty 1}=\underline{\mathrm{y}} \underline{\mathbf{z}}, \hat{\epsilon}_{01}=\mathrm{a} \underline{\mathbf{z}}$, $\hat{\epsilon}_{\infty 2}=\underline{\underline{l}} \underline{\text { n}}$, and $\hat{\epsilon}_{02}=\mathrm{c} \underline{\mathrm{z}}$. Fig. 3 shows the twists of 


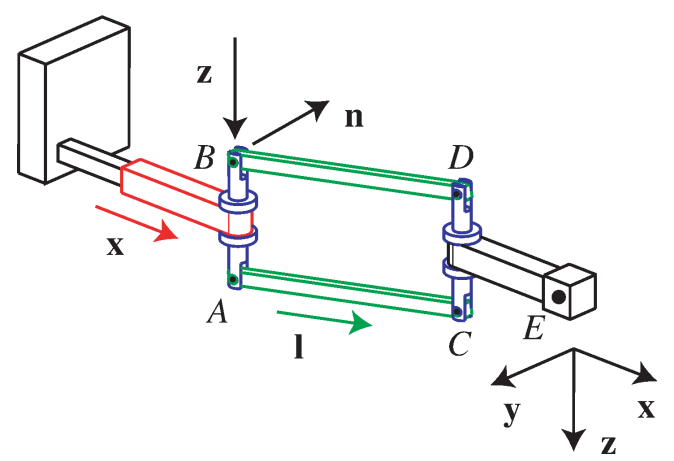

Figure 2: Schematic of PR $\Pi$ R limb

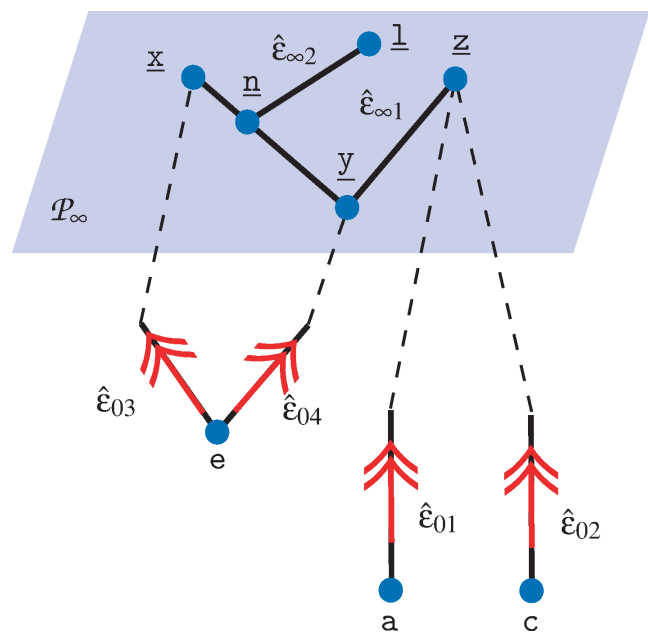

Figure 3: Twists of the PR $\Pi \mathrm{R}$ limb into the projective space

the PR $\Pi \mathrm{R}$ limb into the projective space.

\subsection{Kinematic Jacobian Matrix of the PR $\Pi \mathbf{R}$ Limb}

The PR $\Pi R$ limb twist system is a screw system of dimension 4 that can be written in a matrix form as follows:

$$
\left[\begin{array}{cccc}
\mathbf{0} & \mathbf{z} & \mathbf{0} & \mathbf{z} \\
\mathbf{x} & \mathbf{r}_{A} \times \mathbf{z} & \mathbf{l} \times \mathbf{n} & \mathbf{r}_{C} \times \mathbf{z}
\end{array}\right]
$$

In order to obtain a Jacobian matrix for the PR $\Pi$ $R$ limb, the matrix expressed in (2) requires two additional twists that represent the constrained motions of the limb. Those constrained motions consist of a two-DOF rotational motion including all rotations about axes orthogonal to $\mathbf{z}$ and passing through point $E$, which is the end-effector of the Orthoglide. The two additional twists can be written as follows: $\hat{\epsilon}_{03}=\left[\begin{array}{c}\mathbf{x} \\ \mathbf{r}_{E} \times \mathbf{x}\end{array}\right]$, and $\hat{\epsilon}_{04}=\left[\begin{array}{c}\mathbf{y} \\ \mathbf{r}_{E} \times \mathbf{y}\end{array}\right]$.

\subsection{Superbracket of the PR $\Pi R$ Limb}

Let $e$ is the extensor of step 1 representing the Plücker coordinate vector of the finite point $E$. Accordingly, $\hat{\epsilon}_{03}=\mathrm{e} \underline{\mathrm{x}}$, and $\hat{\epsilon}_{04}=\mathrm{e} \underline{\mathrm{y}}$. As a result, the superbracket of the PR $\Pi \mathrm{R}$ limb twist system is:

$$
S_{\mathrm{PR} \Pi \mathrm{R}}=[\underline{\mathrm{y}} \underline{\mathrm{z}}, \text { a } \underline{\mathrm{z}}, \underline{\mathrm{l}} \underline{\mathrm{n}}, \mathrm{c} \underline{\mathrm{z}}, \text { e } \underline{\mathrm{x}}, \mathrm{e} \underline{\mathrm{y}}]
$$

This superbracket can be expanded into 24 monomials and then reduced to obtain the following simplified expression:

$$
S_{\mathrm{PR} \Pi \mathrm{R}}=[\underline{\mathrm{y}} \underline{\mathrm{z}} \text { a c }][\underline{\mathrm{z}} \underline{\mathrm{l}} \underline{\mathrm{n}} \mathrm{e}][\underline{\mathrm{z}} \underline{\mathrm{x}} \mathrm{e} \underline{\mathrm{y}}]
$$

\subsection{Singularity Conditions of the PR $\Pi R$ Limb}

The singularities of the PR $\Pi \mathrm{R}$ limb correspond to the vanishing conditions of the simplified superbracket decomposition expressed in (4), which happen whenever:

- $[\mathrm{y} \underline{\mathrm{z}} \mathrm{ac}]=0$. Since vector $\mathbf{l}$ is parallel to line $A C$, this condition can be written in a vector form as follows:

$$
(\mathbf{y} \times \mathbf{z}) \bullet \mathbf{l}=0
$$

Equation (5) holds true in each of the following cases:

1. Line $A C$ is parallel to $\mathbf{y}$, which means that vectors $\mathbf{l}$ and $\mathbf{y}$ are parallel to each other. Such a configuration is illustrated in Fig. 4;

2. Line $A C$ is parallel to $\mathbf{z}$, which means that vectors $\mathbf{I}$ and $\mathbf{z}$ are parallel to each other;

3. Vectors $\mathbf{y}$ and $\mathbf{z}$ are parallel to each other. This condition can never be true due to the geometry of the limb;

4. Point $C$ belongs to plane $(A, \mathbf{y}, \mathbf{z})$. 


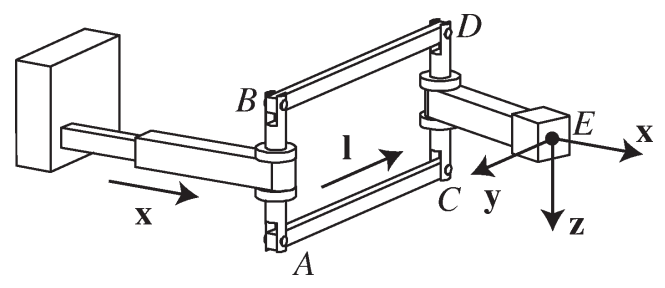

Figure 4: PR $\Pi \mathrm{R}$ limb singularity: line $A C$ is parallel to $\mathbf{y}$

- $[\underline{\mathrm{z}} \underline{\mathrm{l}} \underline{\mathrm{n}} \mathrm{e}]=0$. The vector form of this condition is given by:

$$
(\mathbf{z} \times \mathbf{l}) \bullet \mathbf{n}=0
$$

Equation (6) holds true in each of the following cases:

1. Vectors $\mathbf{I}$ and $\mathbf{z}$ are parallel to each other. This corresponds to the flattening of the parallelogram, as shown in Fig. 5. This configuration of the $\Pi$-joint cannot be reached with the current design of the Orthoglide limb but could be reached with another design of the $\Pi$-joint;

2. Vectors $\mathbf{n}$ and $\mathbf{z}$ are parallel to each other. This condition can never be true due to the geometry of the limb;

3. Vectors $\mathbf{l}, \mathbf{n}$, and $\mathbf{z}$ are linearly dependent, which means that those three vectors are parallel to a same plane.

- $[\underline{z} \underline{x}$ e $\underline{y}]=0$. The vector form of this condition is given by:

$$
(\mathbf{z} \times \mathbf{x}) \bullet \mathbf{y}=0
$$

Clearly, this condition is impossible.

\section{Singularity Analysis of a 5-DOF RUU limb}

\subsection{4-RUU Parallel Manipulator}

The 4-RUU PM [10] shown in Fig. 6 is a 4-DOF $\mathrm{PM}$ whose moving platform performs a 3T1R motion, the rotation being about an axis directed along z. The 4-RUU PM assembly and architecture require that the fifth revolute joint in each limb remains parallel to $\mathbf{z}$ unless the 4-RUU PM reaches a specific

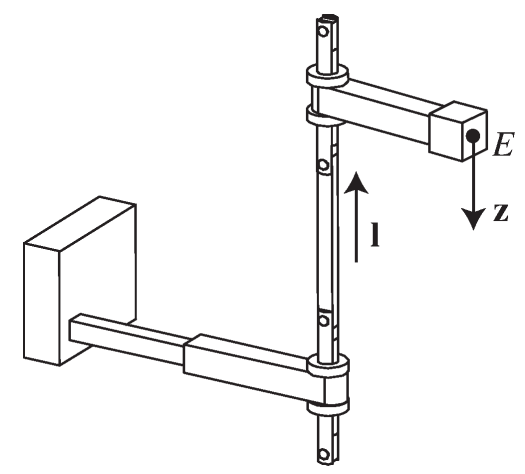

Figure 5: PR $\Pi \mathrm{R}$ limb singularity: vectors $\mathbf{l}$ and $\mathbf{z}$ are parallel to each other

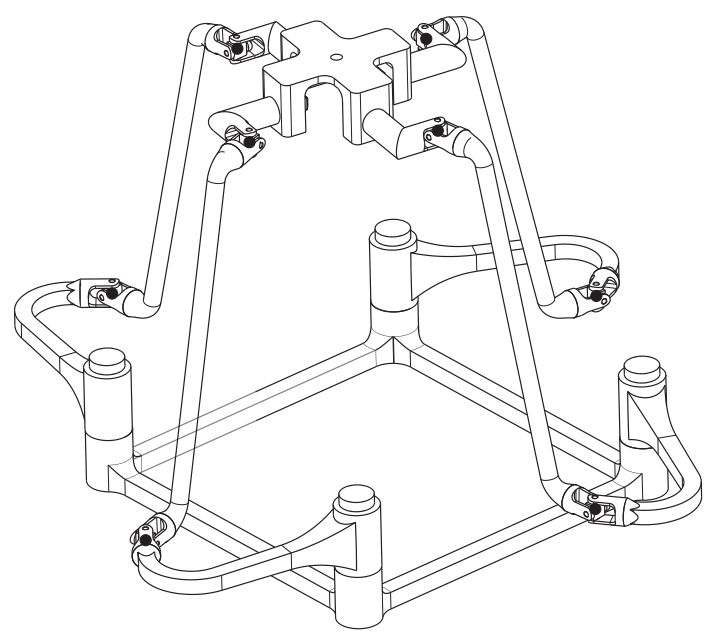

Figure 6: 4-RUU parallel manipulator

parallel singularity called constraint singularity [16]. Here, we focus on the enumeration of the RUU limb singularities. Each RUU limb is a $3 \mathrm{~T} 2 \mathrm{R}$ limb, i.e., provides a three independent translations and two independent rotations.

\subsection{RUU Limb Description}

Fig. 7 shows a 5-DOF limb composed as follows. The first revolute joint axis is parallel to vector $\mathbf{z}$ of fixed direction and passes through point $A$. The second revolute joint axis is also parallel to $\mathbf{z}$ and passes through point $B$. The third revolute joint axis is parallel to vector $\mathbf{m}$ and passes through point $B$. The fourth revolute joint axis is parallel to $\mathbf{m}$ and passes through point $C$. Finally, the axis of the fifth revolute joint is parallel to $\mathbf{z}$ and passes through point $C$. Note that $\mathbf{z}$ and $\mathbf{m}$ are orthogonal to each other. Fur- 


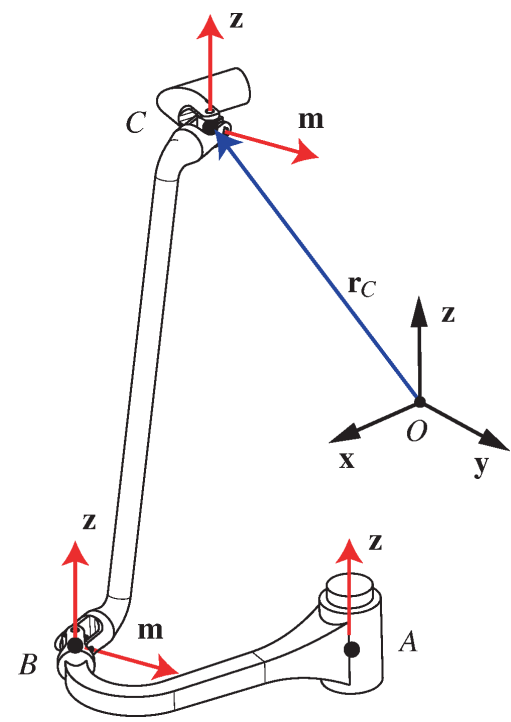

Figure 7: Schematic of RUU limb

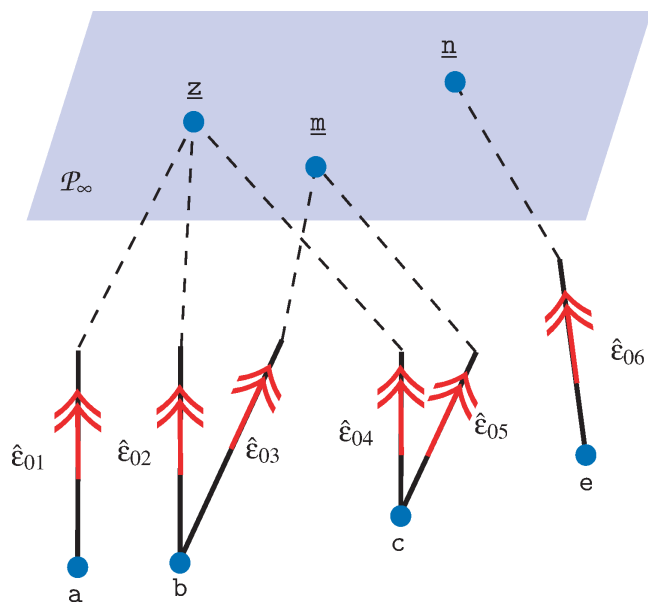

Figure 8: Twists of the RUU limb into the projective space

thermore, the axes of the second and third revolute joints, and similarly, the axes of the fourth and fifth revolute joints intersect and are perpendicular to each other, thus forming a universal joint.

According to the above description, the RUU limb twists are: $\hat{\epsilon}_{01}=\left[\begin{array}{c}\mathbf{z} \\ \mathbf{r}_{A} \times \mathbf{z}\end{array}\right], \hat{\epsilon}_{02}=\left[\begin{array}{c}\mathbf{z} \\ \mathbf{r}_{B} \times \mathbf{z}\end{array}\right], \hat{\epsilon}_{03}=$ $\left[\begin{array}{c}\mathbf{m} \\ \mathbf{r}_{B} \times \mathbf{m}\end{array}\right], \hat{\epsilon}_{04}=\left[\begin{array}{c}\mathbf{m} \\ \mathbf{r}_{C} \times \mathbf{m}\end{array}\right]$, and $\hat{\epsilon}_{05}=\left[\begin{array}{c}\mathbf{z} \\ \mathbf{r}_{C} \times \mathbf{z}\end{array}\right]$.

\subsection{RUU Limb Twist System}

Let $\mathrm{a}, \mathrm{b}$, and $\mathrm{c}$ be the extensors representing the the finite points $A, B$, and $C$, respectively. In addition, let $\underline{\mathbf{z}}=\left[\begin{array}{l}\mathbf{z} \\ 0\end{array}\right]$, and $\underline{\mathbf{m}}=\left[\begin{array}{c}\mathbf{m} \\ 0\end{array}\right]$ represent the points at infinity of any finite line along vectors $\mathbf{z}$, and $\mathbf{m}$, respectively. Thus, $\hat{\epsilon}_{01}=\mathrm{a} \underline{\mathbf{z}}, \hat{\epsilon}_{02}=\mathrm{b} \underline{\mathbf{z}}, \hat{\epsilon}_{03}=\mathrm{b} \underline{\underline{m}}$, $\hat{\epsilon}_{04}=\mathrm{c} \underline{\mathrm{m}}$, and $\hat{\epsilon}_{05}=\mathrm{c} \underline{\mathrm{z}}$. The twists of the RUU limb are represented in the projective space in Fig. 8.

\subsection{Kinematic Jacobian Matrix of the RUU Limb}

The RUU limb twist system is a screw system of dimension 5 that can be written in a matrix form as follows:

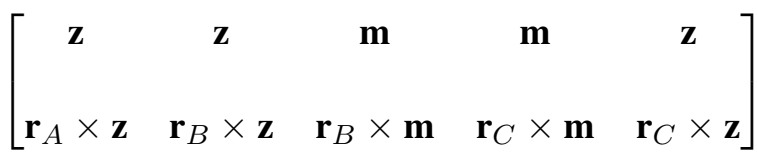

According to Section 3.2, the Jacobian matrix for the RUU limb is constructed by adding, to the matrix obtained in (8), one twist that corresponds to the constrained motion of the limb. This motion is a rotation about an axis directed along $\mathbf{z} \times \mathbf{m}$ and passing through point $E$, which is the end-effector of the 4RUU PM. The additional twist is thus a zero-pitch twist $\hat{\epsilon}_{06}=\left[\begin{array}{c}\mathbf{z} \times \mathbf{m} \\ \mathbf{r}_{E} \times(\mathbf{z} \times \mathbf{m})\end{array}\right]$.

\subsection{Superbracket of the RUU Limb}

Let e be the extensor of step 1 representing the Plücker coordinate vector of the finite point $E$ and let $\underline{\underline{n}}=\left[\begin{array}{c}\mathbf{z} \times \mathbf{m} \\ 0\end{array}\right]$ be the point at infinity of any finite line along vector $\mathbf{z} \times \mathbf{m}$. Accordingly, $\hat{\epsilon}_{06}=\mathrm{e} \underline{\mathrm{n}}$ and the superbracket of the RUU limb twist system can be expressed as follows:

$$
S_{\mathrm{RUU}}=[\mathrm{a} \underline{\mathrm{z}}, \mathrm{b} \underline{\mathrm{z}}, \mathrm{b} \underline{\mathrm{m}}, \mathrm{c} \underline{\mathrm{m}}, \mathrm{c} \underline{\mathrm{z}}, \mathrm{e} \underline{\mathrm{n}}]
$$

This superbracket can be expanded into 24 monomials and then reduced to obtain the following simplified expression:

$$
S_{\mathrm{RUU}}=[\mathrm{a} \underline{\mathrm{z}} \mathrm{bc}][\underline{\mathrm{z}} \mathrm{b} \underline{\mathrm{m}} \mathrm{c}][\underline{\mathrm{z}} \underline{\mathrm{m}} \mathrm{e} \underline{\mathrm{n}}]
$$




\subsection{Singularity Conditions of the RUU Limb}

The singularities of the RUU limb correspond to the vanishing conditions of the simplified superbracket decomposition expressed in (10), which happen whenever:

- $[a \underline{z} b c]=0$. This condition occurs iff the projective points $\mathrm{a}, \underline{\mathbf{z}}, \mathrm{b}$, and $\mathrm{c}$ are coplanar. In the real space, this condition can be written in a vector form as follows:

$$
\left(\mathbf{u}_{A B} \times \mathbf{u}_{A C}\right) \bullet \mathbf{z}=0
$$

where $\mathbf{u}_{A B}$ and $\mathbf{u}_{A C}$ are the unit vectors along line $A B$ and $A C$, respectively. Equation (11) holds true in each of the following cases:

1. Points $A, B$, and $C$ are aligned;

2. Line $A B$ is parallel to $\mathbf{z}$. This condition can never be true due to the geometry of the limb;

3. Line $A C$ is parallel to $\mathbf{z}$. This condition is illustrated in Fig. 9;

4. Line $B C$ is parallel to $\mathbf{z}$;

5. Vector $\mathbf{z}$ is parallel to plane $A B C$.

- $[\underline{z} \mathrm{~b} \underline{\mathrm{m}} \mathrm{c}]=0$. This condition occurs iff the projective points $\underline{z}, \mathrm{~b}, \underline{\mathrm{m}}$, and $\mathrm{c}$ are coplanar. In the real space, this limb singularity can be written in a vector form as follows:

$$
\left(\mathbf{u}_{B C} \times \mathbf{z}\right) \bullet \mathbf{m}=0
$$

where $\mathbf{u}_{B C}$ is the unit vector along line $B C$. Equation (12) holds true in each of the following cases:

1. Line $B C$ is parallel to z. Fig. 10 illustrates such a singular configuration;

2. Line $B C$ is parallel to $\mathbf{m}$;

3. Vectors $\mathbf{z}$ and $\mathbf{m}$ are parallel to each other. This condition can never be true due to the geometry of the limb;

4. Point $C$ belongs to plane $(B, \mathbf{z}, \mathbf{m})$.

- $[\underline{z} \underline{m} \in \underline{n}]=0$. In the real space, this limb singularity can be written in a vector form as follows:

$$
(\mathbf{z} \times \mathbf{m}) \bullet \mathbf{n}=0
$$

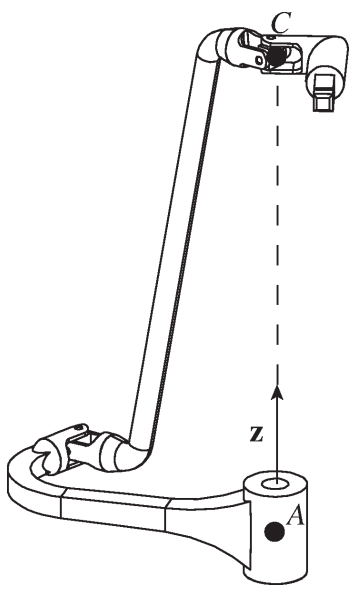

Figure 9: RUU limb singularity: line $A C$ is parallel to $\mathbf{z}$

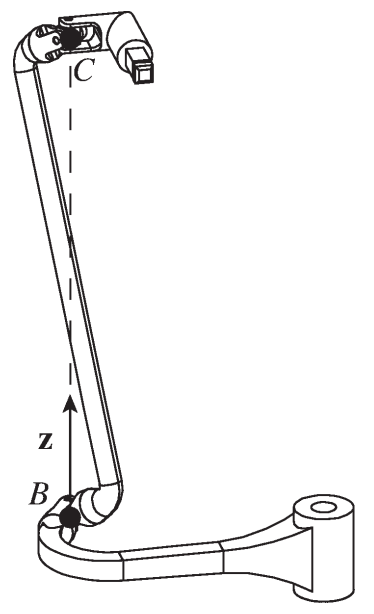

Figure 10: RUU limb singularity: line $B C$ is parallel to $\mathbf{z}$

This is impossible since $\mathbf{n}=\mathbf{z} \times \mathbf{m}$ in any limb configuration.

\subsection{Comparison with Screw-Theory-Based Ap- proach}

In this section, the RUU limb singularities are analyzed using screw theory and the results are compared with those obtained with GCA. The GCA approach consists of extending the $6 \times 5$ matrix of the limb twist system expressed in (8) to obtain a $6 \times 6$ Jacobian matrix and then applying the superbracket decomposition. However, the screw theory approach consists of direct examination of (8) whose rank de- 
ficiency conditions yield the RUU limb singularities. After some linear transformations, (8) becomes:

$\left[\begin{array}{ccccc}\mathbf{z} & \mathbf{m} & \mathbf{0} & \mathbf{0} & \mathbf{0} \\ \mathbf{r}_{A} \times \mathbf{z} & \mathbf{r}_{B} \times \mathbf{m} & \mathbf{u}_{A B} \times \mathbf{z} & \mathbf{u}_{A C} \times \mathbf{z} & \mathbf{u}_{B C} \times \mathbf{m}\end{array}\right]$

The first two columns are zero-pitch twists and correspond to rotational DOFs while the last three ones are infinite-pitch twists and correspond to translational DOFs. Since vectors $\mathbf{m}$ and $\mathbf{z}$ are independent, the above matrix degenerates in configurations where its last three columns are linearly dependent. It can be concluded that in any limb singularity the moving platform of the 4-RUU PM loses translational $\operatorname{DOF}(\mathrm{s})$.

The $\underline{R} U U$ limb exhibits singularity whenever at least one of its three infinite-pitch twists vanishes or whenever at least two of its infinite pitch twists coincide. These conditions occur whenever vectors $\mathbf{u}_{A B} \times \mathbf{z}, \mathbf{u}_{A C} \times \mathbf{z}$, and $\mathbf{u}_{B C} \times \mathbf{m}$ are linearly dependent. Accordingly, the following singularity conditions can be obtained:

1. Points $A, B$, and $C$ are aligned;

2. Line $A B$ is parallel to $\mathbf{z}$;

3. Line $A C$ is parallel to $\mathbf{z}$;

4. Line $B C$ is parallel to $\mathbf{z}$;

5. Line $B C$ is parallel to $\mathbf{m}$.

These conditions, in addition to others, have been obtained with the GCA-based approach in Sec. 5.6. Clearly, GCA-based approach is more systematic as it results in more general results and easily obtaining vector and generic conditions of limb singularities.

\section{Conclusion}

This paper proposed a technique to construct a Jacobian matrix and a superbracket for any lowermobility limb by adding, to the $n$ twists representing the limb twist system, $6-n$ linearly independent twist(s) corresponding to the limb constrained motion(s). Accordingly, a new method for computing lower-mobility limb singularities was introduced. The new method was investigated and applied to four- and five-degrees of freedom limbs and compared with the screw-theory-based approach as well. The proposed method is systematic and leads to an exhaustive enumeration and interpretation of parallel manipulators' limb singularities.

\section{References}

[1] R. Clavel, Delta, a fast robot with parallel geometry. Proceedings of the International Symposium On Industrial Robot, Switzerland, 1988, pp. 91-100.

[2] B. Hu, B. Mao, J. Yu, \& Y. Lu, Unified stiffness model of lower mobility parallel manipulators with linear active legs, International Journal of Robotics and Automation, 29(1), 2014, DOI: 10.2316/Journal.206.2014.1.206-3807.

[3] Y. Xu, J. Yao, \& Y. Zhao, Internal forces analysis of the active overconstrained parallel manipulators, International Journal of Robotics and Automation, 30(5), 2015, DOI: 10.2316/Journal.206.2015.5.206-4422.

[4] L Wang, D Wang, \& J. Wu, A control strategy of a 2-dof parallel manipulator with fractional order PD $\mu$ control, International Journal of Robotics and Automation, 32(4), 2017, DOI: 10.2316/Journal.206.2017.4.206-4861.

[5] Y. Fang, \& L.-W. Tsai, Structure synthesis of a class of 4-DoF and 5-DoF parallel manipulators with identical limb structures. The International Journal of Robotics Research, 21(9), 2002, 799-810.

[6] S.-J. Zhu, \& Z. Huang, Singularity analysis for a 5-DoF fully-symmetrical parallel manipulator 5-RRR(RR). IEEE International Conference on Robotics and Automation, Roma, Italy, 2007.

[7] S. Guo, C. Wang, H. Qu, \& Y. Fang, A novel 4RRCR parallel mechanism based on screw theory and its kinematics analysis. Proceedings of the Institution of Mechanical Engineers, Part C: Journal of Mechanical Engineering Science, 227(9), 2012, 2039-2048. 
[8] P. Ben-Horin, \& M. Shoham, Singularity condition of six-degree-of-freedom three-legged parallel robots based on Grassmann-Cayley algebra, IEEE Transactions on Robotics, 22(4), 2006, 577-590.

[9] D. Kanaan, P. Wenger, S. Caro, \& D. Chablat, Singularity analysis of lower-mobility parallel manipulators using Grassmann-Cayley algebra, IEEE Transactions on Robotics, 25(5), 2009, 995-1004.

[10] S. Amine, M. Tale Masouleh, S. Caro, P. Wenger, \& C. Gosselin, Singularity conditions of 3T1R parallel manipulators with identical limb structures, ASME Journal of Mechanisms and Robotics, 4(1), 2012, 1-11.

[11] T. McMillan, Invariants of antisymmetric tensors, PhD Thesis, University of Florida, Gainesville, Florida, USA, 1990.

[12] K. H. Hunt, Kinematic geometry of mechanisms, Clarendon Press, Oxford, 1978.

[13] X. Kong, \& C. Gosselin, Type synthesis of parallel mechanisms, (Germany: Springer, 2007).

[14] P. Wenger, \& D. Chablat, Kinematics analysis of a new parallel machine-tool: the Orthoglide, Advances in Robot Kinematics, (Netherlands, Dordrecht: Springer, 2000) 305-314.

[15] S. Caro, W. A. Khan, D. Pasini, \& J. Angeles, The rule-based conceptual design of the architecture of serial Schönflies-motion generators, Mechanism and Machine Theory 45(2), 2010, 251-260.

[16] D. Zlatanov, I. A. Bonev, \& C. Gosselin, Constraint singularities of parallel mechanisms, IEEE International Conference on Robotics and Automation, Washington, DC, 2002.

\section{Authors' Information}

Semaan Amine received the B.E. degree in Mechanical Engineering from the Lebanese University, Lebanon, in 2008, and the M.Sc. and Doctorate degrees in Mechanical Engineering from Ecole
Centrale de Nantes, France, in 2008 and 2011, respectively. He is currently and Assistant Professor with the Department of Mechanical Engineering at Beirut Arab University, Beirut, Lebanon. His research interests include: robot kinematics, type synthesis of parallel robots, and vehicle dynamics.

Ossama Mokhiamar received the B.Sc. and M.Sc. degrees in Mechanical Engineering from Alexandria University, Alexandria, Egypt, in 1994 and 1997, respectively and the Ph.D. degree in Mechanical Engineering from Kanagawa Institute of Technology, Japan, in 2005. He is currently an Assistant Professor with the Department of Mechanical Engineering at Alexandria University, Alexandria, Egypt. His research interests include: robot kinematics, nonlinear control, and vehicle dynamics.

Stéphane Caro received the Engineering and M.Sc. degrees in Mechanical Engineering from Ecole Centrale Nantes (ECN), Nantes, France, in 2001, and the Doctorate degree in Mechanical Engineering from the University of Nantes in 2004. $\mathrm{He}$ was a Post-doctoral Fellow in the Centre for Intelligent Machines, McGill University, Montreal, QC, Canada from 2005 to 2006. He was awarded the accreditation to supervise research (HDR) in 2014. He is currently Research Director at the National Centre for Scientific Research (CNRS) and works in the Nantes Digital Science Laboratory (LS2N), UMR CNRS 6004, France. He is the head of the Robots and Machines for Manufacturing Society and Services (ROMAS) team at LS2N. He is also a part-time researcher at IRT Jules Verne, a mutualized industrial research institute. Moreover, he is a lecturer at Ecole Centrale de Nantes and IMT Atlantique Bretagne-Pays de la Loire, France. His research interests include design and modeling of cable-driven parallel robots, conceptual design of parallel robots, robust design, kinematic analysis, singularity analysis and type-synthesis of parallel robots, sensitivity analysis, tolerance synthesis, and design optimization. 